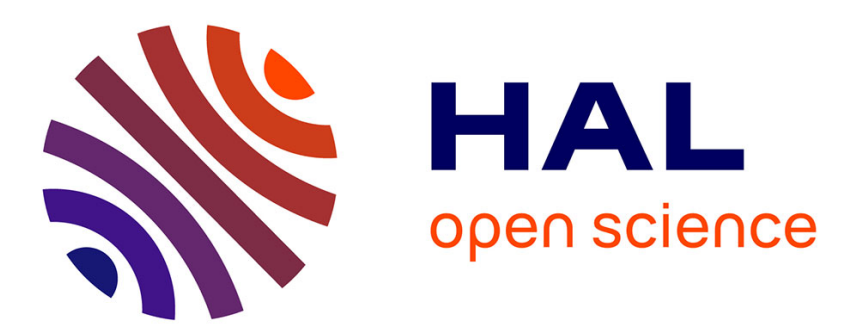

\title{
Estimation of Interaction Forces between Two Magnetic Bolus-like Microrobots
}

\author{
Lyès Mellal, David Folio, Karim Belharet, Antoine Ferreira
}

\section{To cite this version:}

Lyès Mellal, David Folio, Karim Belharet, Antoine Ferreira. Estimation of Interaction Forces between Two Magnetic Bolus-like Microrobots. International Conference on Robotics, Manipulation, and Automation at Small Scales, Jul 2016, Paris, France. pp.1 - 6, 10.1109/MARSS.2016.7561740 . hal-01414182

\section{HAL Id: hal-01414182 \\ https://hal.science/hal-01414182}

Submitted on 12 Dec 2016

HAL is a multi-disciplinary open access archive for the deposit and dissemination of scientific research documents, whether they are published or not. The documents may come from teaching and research institutions in France or abroad, or from public or private research centers.
L'archive ouverte pluridisciplinaire HAL, est destinée au dépôt et à la diffusion de documents scientifiques de niveau recherche, publiés ou non, émanant des établissements d'enseignement et de recherche français ou étrangers, des laboratoires publics ou privés. 


\title{
Estimation of Interaction Forces between Two Magnetic Bolus-like Microrobots
}

\author{
Lyès Mellal $^{1}$, David Folio ${ }^{1}$, Karim Belharet ${ }^{2}$, and Antoine Ferreira ${ }^{1}$
}

\begin{abstract}
This paper analyses the interaction forces between two magnetic boluses for future drug targeting applications. To transport the drugs, it is necessary to convey several therapeutic magnetic boluses using magnetic gradients. The main difficulty is to control a group of different therapeutic boluses at desired states, despite the presence of interaction forces between boluses. To overcome this issue and designing robust control strategies, it is important to fully understand these interactions forces. Based on a dipole-dipole interaction model and dynamic modeling of two magnetic boluses, the magnetic and non magnetic forces are expressed. Finally, an experimental investigation is carried out in a tank under the presence of the magnetic field in order to to assess the prevalence between the magnetic and the non-magnetic interaction forces.
\end{abstract}

\section{INTRODUCTION}

Magnetically actuated microrobots have been proposed for numerous applications, as their small scales enable the access to complex environments [1], [2], [3]. Especially, microrobots are applied in minimally-invasive surgery (MIS) procedure, including: targeted drug delivery, brachytherapy, hyperthermia, removing material by mechanical means or acting as simple static structures [4], [5], [6], [7]. These microrobots are commonly referred as therapeutic micro carriers (TMMC) [8], [9]. To embed the therapeutic agent, the TMMC could be either magnetic helical medical microrobots [10], [11], magnetic microbeads [12], [13], or micro/nano-particles suspended in a carrier fluid (ie. a ferrofluid) coated with organic polymer to prevent agglomeration and improve surface functionality [9], [14], [15]. Then, an external magnetic field is used to steer the TMMCs along a pre-planned path to the targeted location [8], [13]. This targeting approach is also able to steer superparamagnetic iron oxide (SPIO) particles using an improved magnetic resonance imaging (MRI) system [8], [9], [16]. To convey the desired amount of drugs, multiple boluses have to be administered, and controlled [17]. Previous studies have considered the magnetic control of a group of millimeter-sized beads immersed in fluid and driven thanks to a MRI scanner [18]. In [19] the authors have investigated the control of geometrically dissimilar Mag- $\mu$ Bots and a group of identically-fabricated microrobots. The authors proved through simulation results the stability of two millimeter-sized beads at a desired positions. However, the understanding of the complete dynamics of several microrobots remains challenging [18]. In particular, the interaction forces acting between

\footnotetext{
${ }^{1}$ L. Mellal, D. Folio and A. Ferreira are with INSA Centre Val de Loire, Université d'Orléans, PRISME EA 4229, Bourges, France. Corresponding author: Antoine Ferreira (Email: antoine.ferreira@insa-cvl.fr

${ }^{2}$ K.Belharet is with Hautes Études d'Ingénieur campus Centre, PRISME EA 4229, Châteauroux, France.
}

multiple microrobots are not fully addressed. This knowledge could greatly improve the design of robust to control law that takes into account this disturbance on the system. This study proposes to investigate these interactions forces between two microrobots. More precisely, superparamagnetic iron oxide (SPIO) particles suspended in non-magnetizable medium, termed hereafter magnetic bolus, is considered. Hence, based on a dipole-dipole interaction model, the dynamic modeling of magnetic in fluidic environment is carried out. Specifically, the magnetic and non-magnetic interaction forces between two boluses in the presence of an external magnetic field $\mathbf{b}_{0}$ is expressed. This paper is divided in four sections. Section II details the mathematical modeling of dipole-dipole interactions in order to model the magnetic interaction forces between two boluses. Then, in Section III, experiments are conducted to estimate the magnetic and non-magnetic interaction forces. Conclusion and discussions on open issues are summarized in Section IV.

\section{MATERIALS AND METHODS}

\section{A. Soft Magnetic bolus Modeling}

In this study, a colloidal suspension of superparamagnetic iron oxide (SPIO) particles is used as magnetic bolus. Hence, the considered magnetic microrobot is a ferrofluid droplet immersed in non-magnetizable medium. Each SPIO particles of the magnetic microrobot carry a magnetic moments. Without external magnetic field $(\mathbf{b}=0)$ their dipole directions are randomly spread, as illustrated in Figure 1(a). In such situation, the ferrofluid droplet adopts commonly a spherical shape to minimize the surface energy. In presence of an applied magnetic field density $\mathbf{b}$, the overall SPIO particles are polarized, and their magnetic moment are mainly aligned with b. Classically, the magnetic bolus takes the shape of an ellipsoid, as depicted in Figure 1(b)-(c).

Commonly, for hard magnetic materials the magnetization $\mathbf{m}$ is independent of the magnetic field $\mathbf{b}$, and could be considered easily saturated in many cases. In contrast, for soft-magnetic materials, as with using SPIO particles $\mathbf{m}$ is strongly related to the field b. At low magnetic fields, such that $|\mathbf{m}|<m_{\text {sat }}$ (with $m_{\text {sat }}$ the saturation magnetization of the material), the magnetization of SPIO particles exhibits typically the following linear behavior [20]:

$$
\mathbf{m}=\frac{\chi_{a}}{\mu_{0}(1+\chi)} \mathbf{b}
$$

where $\mu=\mu_{0}(1+\chi)$ is the permeability of the bolus, and $\chi_{a} \in \Re^{3}$ is the apparent susceptibility tensor that is related to the bolus shape. Here, the considered magnetic bolus varies 


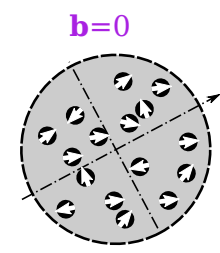

(a)

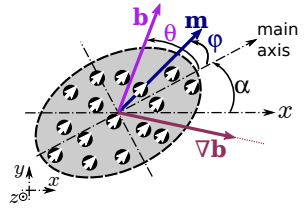

(b)

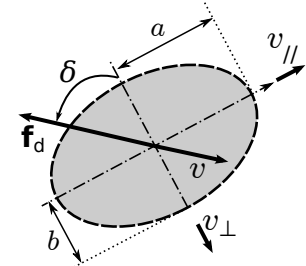

(c)
Figure 1. Soft-magnetic microrobot: (a) without external magnetic field; and (b) with an external magnetic field $\mathbf{b}_{0}$ and gradient $\nabla \mathbf{b}$ leading to an ellipsoidal ferrofluid arrangement. The black circles depict the SPIO particles with their magnetic moments $\mathbf{m}$ shown with the white arrows. (c) Schematic model of a prolate ellipsoid of revolution $(a \geq b=c)$ with $a$ the major semiaxis and $b$ the minor semiaxis..

from spherical geometry to axisymmetric prolate ellipsoid (see Fig. 1(c)). For spheroid the susceptibility tensor is expressed as follows [20]:

$$
\chi_{a}=\operatorname{diag}\left(\frac{\chi}{1+n_{a} \chi}, \frac{\chi}{1+n_{b} \chi}, \frac{\chi}{1+n_{b} \chi}\right)
$$

where $n_{a}$ and $n_{b}$ are demagnetizing factors along the major and minor axis, given by [20], [21]:

$$
\begin{aligned}
1 & =n_{a}+2 n_{b} \\
n_{a} & =\frac{1-\varepsilon^{2}}{2 \varepsilon^{3}}\left(\log \left(\frac{1+\varepsilon}{1-\varepsilon}\right)-2 \varepsilon\right)
\end{aligned}
$$

with $\varepsilon=\sqrt{1-\Lambda^{-2}}$ the eccentricity, and $\Lambda=a / b$ the aspect ratio of the prolate ellipsoidal magnetic bolus (see Fig. 1(c)). In the case of spherical device the demagnetizing factors simplify to $n_{a}=n_{b}=1 / 3$. From the above equations, the magnetization $\mathbf{m}=\left(m_{x}, m_{y}, m_{z}\right)^{T}$ is then related to the magnetic field $\mathbf{b}=\left(b_{x}, b_{y}, b_{z}\right)^{T}$ as follows:

$$
\left(\begin{array}{l}
m_{x} \\
m_{y} \\
m_{z}
\end{array}\right)=\frac{V_{m} \chi}{\mu_{0}(1+\chi)}\left(\begin{array}{c}
\frac{b_{x}}{1+n_{a} \chi} \\
\frac{b_{y}}{1+n_{b} \chi} \\
\frac{b_{z}}{1+n_{b} \chi}
\end{array}\right)
$$

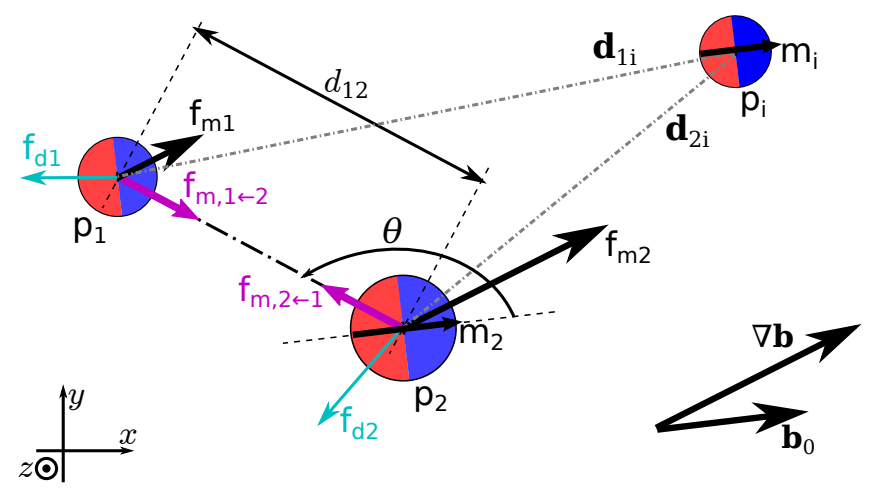

Figure 2. Group of magnetic microbeads in a microfluidic environment.

Multiple magnetic boluses have to be serially administered and controlled. A key issue in the success of the operation is then to address the interactions forces involved in their dynamic behavior. Especially, within a magnetic field each boluses induce a dipole-dipole interaction force. It is important to study this dipole-dipole interaction force.

\section{B. Dipole-dipole Interaction Forces}

Commonly, when several magnetic particles are in a closed range, a magnetic interaction occurs. Specifically, a magnetic bolus $i$ located at $\mathbf{p}_{i}=\left(x_{i}, y_{i}, z_{i}\right)^{T}$ with its dipole moment ${ }^{i} \mathbf{m}$ induces a magnetic field in location $\mathbf{p}$ given by [22], [23]:

$$
\mathbf{b}_{\mathbf{p}_{i}}(\mathbf{p})=\frac{\mu_{0}}{4 \pi}\left(3\left({ }^{i} \mathbf{m} \cdot \mathbf{d}_{0}\right) \mathbf{d}_{0}-{ }^{i} \mathbf{m}\right)
$$

where $\mu_{0}=4 \pi \times 10^{-7} \mathrm{Tm} / \mathrm{A}$ is the vacuum permeability; $\mathbf{d}_{0}=\frac{\left(\mathbf{p}-\mathbf{p}_{i}\right)}{\left\|\mathbf{p}-\mathbf{p}_{i}\right\|}$ is the separation distance unit vector (see also Fig. 2). Thus, the interaction force acting on the magnetic particle 2 with dipole moment ${ }^{2} \mathbf{m}$ due to the presence of a particle 1 with dipole moment ${ }^{1} \mathbf{m}$ is expressed as [24]:

$$
\begin{aligned}
\mathbf{f}_{\mathrm{im}, 2 \leftarrow 1}=\frac{3 \mu_{0}}{4 \pi d_{12}^{4}} & \left(\mathbf{d}\left({ }^{1} \mathbf{m} \cdot{ }^{2} \mathbf{m}\right)+{ }^{1} \mathbf{m}\left(\mathbf{d}_{12} \cdot{ }^{2} \mathbf{m}\right)\right. \\
& +{ }^{2} \mathbf{m}\left(\mathbf{d}_{12} \cdot{ }^{1} \mathbf{m}\right) \\
& \left.-5 \frac{\mathbf{d}}{d_{12}^{2}}\left(\mathbf{d}_{12} \cdot{ }^{2} \mathbf{m}\right)\left(\mathbf{d}_{12} \cdot{ }^{1} \mathbf{m}\right)\right)
\end{aligned}
$$

where $\mathbf{d}_{12}=d_{12} \mathbf{d}_{0}$ is the separation distance vector between the two particles, as illustrated in Fig. 2. Obviously, the Newton's third law implies: $\mathbf{f}_{\mathrm{im}, 1 \leftarrow 2}=-\mathbf{f}_{\mathrm{im}, 2 \leftarrow 1}$. Then, the magnetic interaction force magnitude between the particles 1 and 2 is defined as :

$$
\begin{aligned}
{ }^{12} \mathbf{f}_{\mathrm{im}} & =\left\|\mathbf{f}_{\mathrm{im}, 1 \leftarrow 2}\right\|=\left\|\mathbf{f}_{\mathrm{im}, 2 \leftarrow 1}\right\| \\
& =\frac{3 \mu_{0}\left\|\mathbf{m}_{1} \mathbf{m}_{2}\right\|}{4 \pi d^{4}} \sqrt{1-2 \cos ^{2} \theta_{12}+5 \cos ^{4} \theta_{12}}
\end{aligned}
$$

with $\theta_{12}$ the angle between the dipole moment $\mathbf{m}_{j}(\mathrm{j}=1$ or 2$)$ and the separation distance direction $\mathbf{d}_{12}$ (see Fig. 2).

First, we assumed that all dipole moments of the magnetic boluses are aligned along the uniform field $\mathbf{b}_{0}$ and are not saturated to their maximum value $\mathbf{m}_{j \text {,sat }}$. Secondly, for a given separation distance $d_{12}$ the above interaction force magnitude is minimal for $\theta_{\min }=k 180^{\circ}+\left\{63.43^{\circ} ; 116.56^{\circ}\right\}$, and maximal for $\theta_{\max }=k 180^{\circ}+\left\{0 ; 180^{\circ}\right\}, \forall k \in \mathbb{Z}$. Commonly, the magnetic gradient is limited by the capability of the magnetic coils system. Hence, the minimal controllable separation distance is given by:

$$
d_{\min }=\left(\frac{6 \mu_{0}\left\|\mathbf{m}_{a} \mathbf{m}_{b}\right\|}{4 \pi\left\|\mathbf{f}_{\text {max }}\right\|}\right)^{1 / 4}
$$

with $\left\|\mathbf{f}_{\mathrm{m}, \text { max }}\right\|$ the maximum magnetic force induced by the magnetic coils system.

Let us consider two magnetic boluses with different radius, $r_{1}=1075 \mu \mathrm{m}, r_{2}=805 \mu \mathrm{m}$. The magnetic interaction force $\mathbf{f}_{\mathrm{im}}$ is computed for different magnetic field $\mathbf{b}$ strengths which is used to magnetize the boluses. As shown in the Fig. 3, the magnetic interaction forces is in the order of micro-newtons for a separation distances in the range of $[3.5 \mathrm{~mm} ; 7 \mathrm{~mm}]$. For a separation distance $d=3.5 \mathrm{~mm}$, the magnetic interaction 
force is about $1.7 \mu \mathrm{N}$ of magnitude when an external magnetic field of $\left\|\mathbf{b}_{0}\right\|=35 \mathrm{mT}$ is applied.

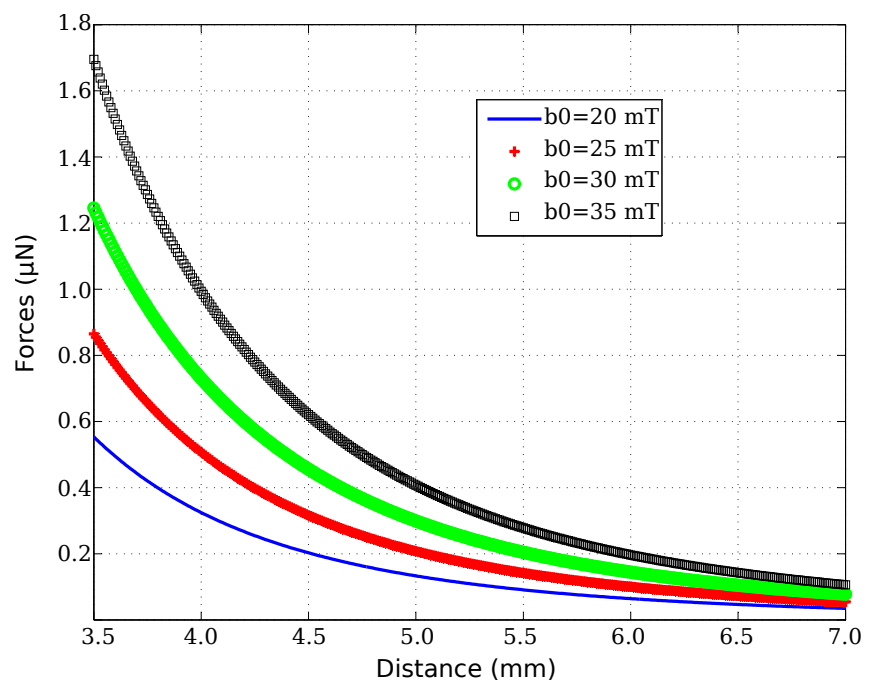

Figure 3. The magnetic interaction force of the bolus as function a separation distance.

The magnetic interaction forces have been theoretically determined. It remains to investigate the other interaction forces acting between boluses.

\section{Total Interaction Forces}

The considered magnetic boluses are ferrofluid droplet immersed in a microfluidic environment. As previously presented, in the presence of a magnetic field, the boluses moved through the magnetic interaction force $\mathbf{f}_{\mathrm{im}}$ acting between them. Other non-magnetic interaction forces also appear, and the total interaction forces could be formulated as follows:

$$
\begin{aligned}
& { }^{12} \mathbf{f}_{i}={ }^{12} \mathbf{f}_{\mathrm{im}}+{ }^{12} \mathbf{f}_{\mathrm{in}} \\
& { }^{21} \mathbf{f}_{i}={ }^{21} \mathbf{f}_{\mathrm{im}}+{ }^{21} \mathbf{f}_{\mathrm{in}}
\end{aligned}
$$

where $f_{i n}$ embeds all other non-magnetic interaction force (non-contact force). Moreover, when magnetic boluses navigate in a microfluidic environment they are subject to the hydrodynamic drag force $f_{d}$. Considering a low motion (that is at a low Reynold number), these hydrodynamic drag forces are estimated from the Stoke's law:

$$
{ }^{i} \mathbf{f}_{\mathrm{d}}=-6 \pi \eta_{f} r_{i} \mathbf{v}_{i}
$$

with $\eta_{f}$ the fluid viscosity; $r_{i}$ and $\mathbf{v}_{i}$ respectively the radii and velocities of the $i$ th magnetic bolus. Thus, using the second principe of Newton's law implies the following dynamic on two boluses:

$$
\begin{aligned}
& m_{1} \ddot{\mathbf{x}}_{1}={ }^{1} \mathbf{f}_{\mathrm{d}}+{ }^{12} \mathbf{f}_{i} \\
& m_{2} \ddot{\mathbf{x}}_{2}={ }^{2} \mathbf{f}_{\mathrm{d}}+{ }^{21} \mathbf{f}_{i}
\end{aligned}
$$

where $\ddot{\mathbf{x}}_{i}$ and $m_{i}$ are the acceleration and the mass of the $i$ th bolus. Therefore, from (10) and (12), the total interaction forces could be estimated from:

$$
\begin{aligned}
&{ }^{12} \mathbf{f}_{i}=m_{1} \ddot{x}_{1}-{ }^{1} \mathbf{f}_{\mathrm{d}} \\
&{ }^{21} \mathbf{f}_{i}=m_{2} \ddot{x}_{2}-{ }^{2} \mathbf{f}_{\mathrm{d}}
\end{aligned}
$$

\section{EXPERIMENTAL RESULTS}

\section{A. Experimental setup}

To estimate experimentally the magnetic interaction forces between two boluses experiments have conducted on our experimental testbed [25]. The experimental platform comprise an electromagnetic actuation (EMA) system developed by Aeon Scientific ${ }^{\mathrm{TM}}$. The EMA system consists of three nested sets of Maxwell coils and one nested set of Helmholtz coils [25], and is illustrated in Fig.4(a). Such arrangement allows generating a constant-gradient magnetic field pointing in $x, y$, and $z$-axis directions. The generated uniform magnetic field strength is limited to $\left\|\mathbf{b}_{\max }\right\|=30 \mathrm{mT}$. Magnetic field will thus be exerted to magnetize the different magnetic boluses that are placed inside a tank filled with an aqueous solution of $50 \%$ glycerin that is closed to the blood viscosity Fig.4(b). The Table I summarizes the relevant experimental parameters set used for the magnetic boluses.

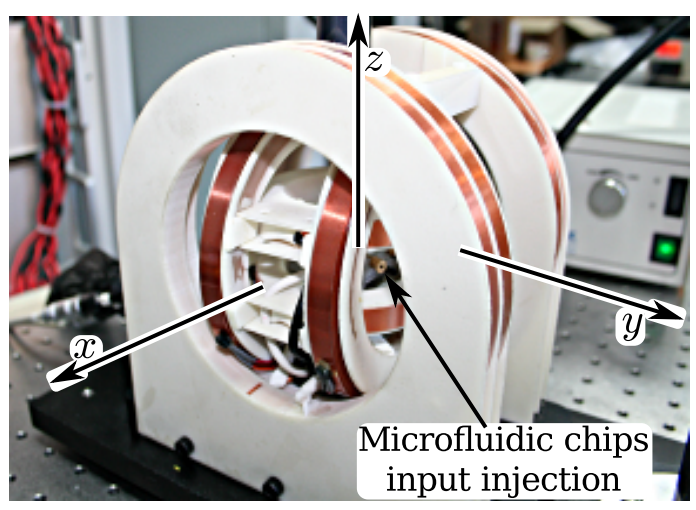

(a)

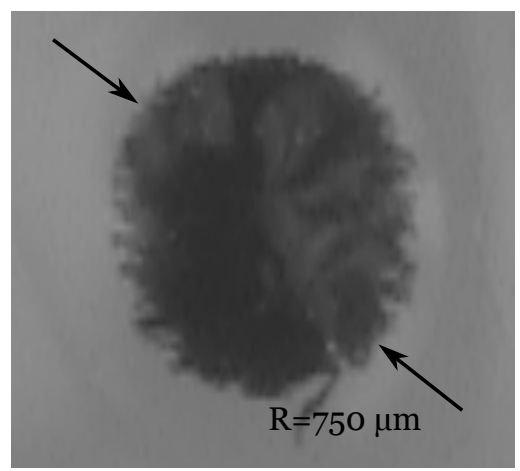

(b)

Figure 4. Experimental setup: (a) 3D Maxwell-Helmholtz coils and (b) a bolus in a tank.

\section{B. Magnetic Bolus Preparation}

The magnetic microrobot is a colloidal suspension of superparamagnetic iron oxide particles (SPIO). More precisely, the ferrofluid is a heterogeneous mixture consisting of a colloidal liquid where magnetite particles $\left(\mathrm{Fe}_{3} \mathrm{O}_{4}\right)$ are suspended in a carrier fluid, that will be in the end replaced with the therapeutic agent. Therefore, two phases can be 
Table I

EXPERIMENTAL PARAMETERS SET

\begin{tabular}{c|cl} 
Parameters & \multicolumn{2}{|c}{ Values } \\
\hline$\chi$ & 0.901 & \\
$\tau_{m}$ & 0.6 & \\
$\rho_{f}$ & 3488 & $\left(\mathrm{~kg} / \mathrm{m}^{3}\right)$ \\
$\rho_{f / b}$ & 920 & $\left(\mathrm{~kg} / \mathrm{m}^{3}\right)$ \\
$\rho_{p}$ & 5242 & $\left(\mathrm{~kg} / \mathrm{m}^{3}\right)$ \\
$r_{1}$ & 1075 & $(\mu \mathrm{m})$ \\
$r_{2}$ & 805 & $(\mu \mathrm{m})$ \\
$\eta_{f}$ & 4 & $(\mathrm{mPas})$ \\
$\eta_{f / b}$ & 62 & $(\mathrm{mPas})$ \\
\hline
\end{tabular}

differentiated: the carrier phase and the surrounding liquid. To mimic different types of drug load, here, SPIO powder (Inoxia Ltd, UK) are suspended in oleaginous phase, and is shown on the Fig.5(a). Sunflower oil, which is biocompatible, biodegradable, environmentally friendly, was selected as the oleaginous carrier liquid in the present work. Thus, the bolus is an homemade ferrofluid that is prepared by mixing sunflower seed oil and the SPIO powder for $30 \mathrm{~min}$ in a volumetric ratio of $\tau_{m}=60 \%$ magnetic particles. This chosen $\tau_{m}$ ratio is related to the optimal magnetization rate that allows conveying an optimum drug load [17]. Then the density of the magnetic boluses is basically computed from:

$$
\rho_{b}=\left(\tau_{m} \rho_{p}+\rho_{f / b}\left(1-\tau_{m}\right)\right)
$$

with $\tau_{m}$ the magnetization rate; $\rho_{p}$ the density of the SPIO $\left(\mathrm{Fe}_{3} \mathrm{O}_{4}\right)$ particles; $\rho_{f / b}$ the ferrofluid carrier fluid density (see also Table I).

\section{Magnetic interaction force estimation}

We evaluated experimentally the magnetic interaction forces between two boluses along a similar direction (x-axis) in a tank filled with an aqueous solution of $50 \%$ glycerin. As shown in Fig. 5(a), the initial distance separating the boluses is settled to $d=6.75 \mathrm{~mm}$ where the magnetic attractive forces could be neglected. Then, each magnetic bolus experiences the same magnetic field $\mathbf{b}=(30 \mathrm{mT}, 0,0)^{T}$. The bolus $B_{1}$ and $B_{2}$ move from their initial positions $x_{1}=-1.7 \mathrm{~mm}, x_{2}=$ $5.05 \mathrm{~mm}$ respectively to the final positions $x_{1 f}=0.78 \mathrm{~mm}$, $x_{2 f}=4.34 \mathrm{~mm}$ depicted in Fig. 5(b). As expected, this one dimensional motion is caused by the presence of an interaction force acting between the two boluses.

As shown in Fig.6, the separation distance between the boluses decreases as function the time until reaching approximately $3.5 \mathrm{~mm}$ which corresponds to the collision between the boluses (cf. Fig.5(b)). The experimental magnetic interaction force acting between boluses is given in Fig.7. A maximal value of $1.2 \mu \mathrm{N}$ is obtained, just before contact between the boluses. Obviously, this magnetic interaction force decreases as a separation distance increases.

The measured mean velocities of boluses are $\mathbf{v}_{1}=$ $1.036 \mathrm{~mm} / \mathrm{s}$ and $\mathbf{v}_{2}=-2.54 \mathrm{~mm} / \mathrm{s}$; and the corresponding accelerations $\ddot{\mathbf{x}}_{1}=-0.0083 \mathrm{~mm} / \mathrm{s}^{2}$ and $\ddot{\mathbf{x}}_{2}=0.0979 \mathrm{~mm} / \mathrm{s}^{2}$. The magnetic boluses interaction forces strength is estimated

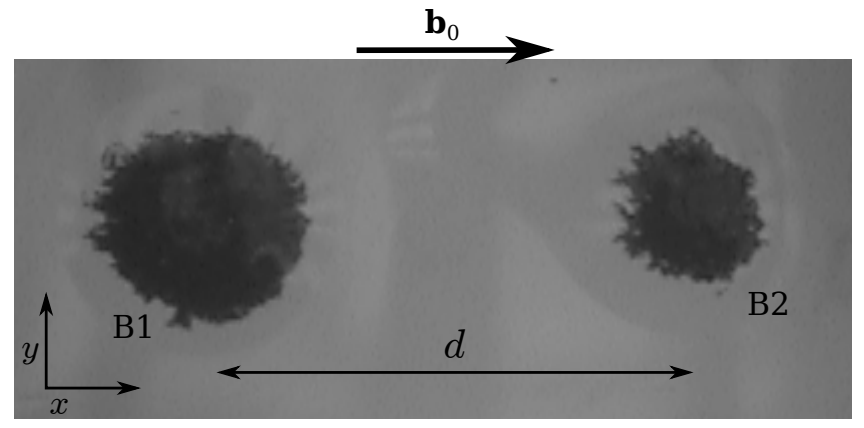

(a)

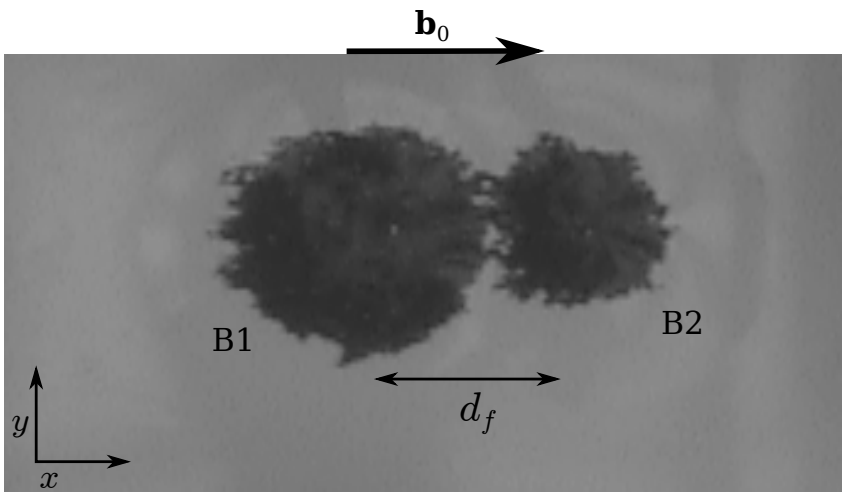

(b)

Figure 5. Two magnetic boluses immersed into a Water/Glycerol tank: (a) the boluses are in their initial positions, (b): the boluses are in collision after application of magnetic field $b_{0 x}=30 \mathrm{mT}$.

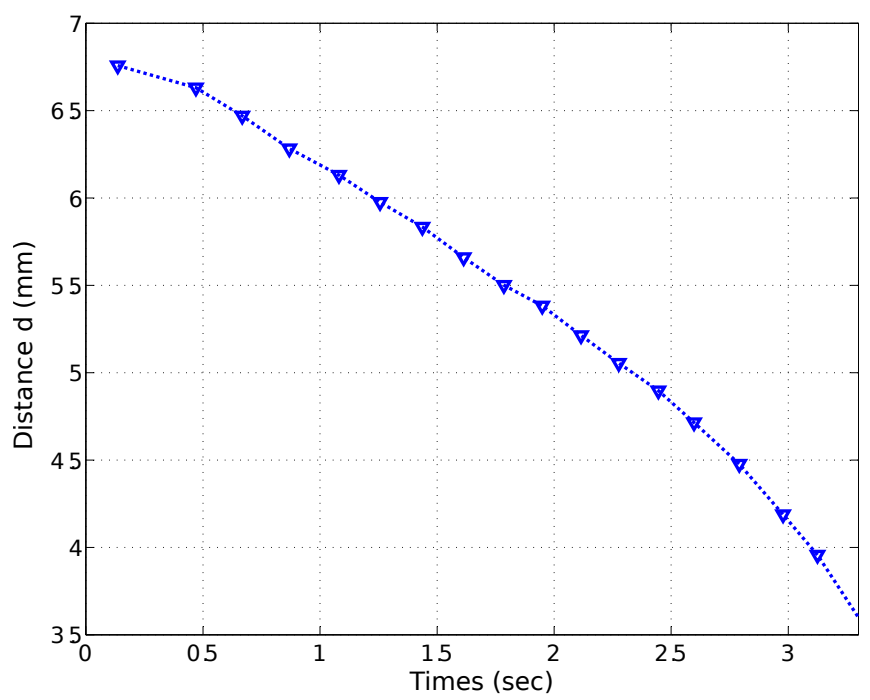

Figure 6. Evolution of separation distance $d$ as function the time.

about ${ }^{12} \mathbf{f}_{i}=0.9435 \mu \mathrm{N}$ and ${ }^{21} \mathbf{f}_{i}=3.0904 \mu \mathrm{N}$. The nonmagnetic interaction forces given in Fig. 8 are in the order of few micro-newton. Therefore, these non-magnetic forces are not negligible [26]. Especially, these interaction forces increase with the boluses' separation distance $d$. 


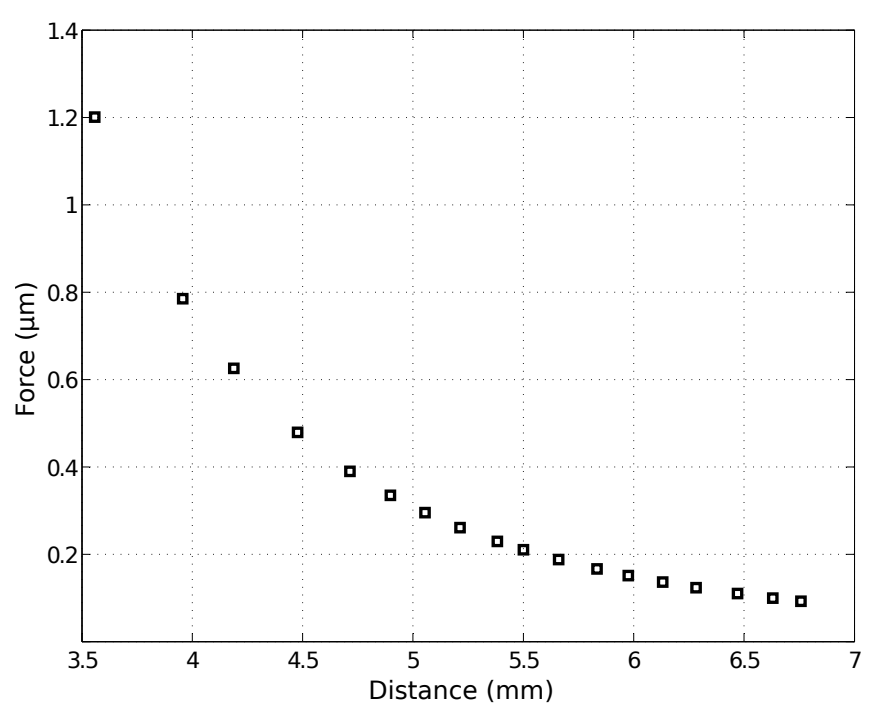

Figure 7. The experimental magnetic interaction force between boluses as function of the separation distance.

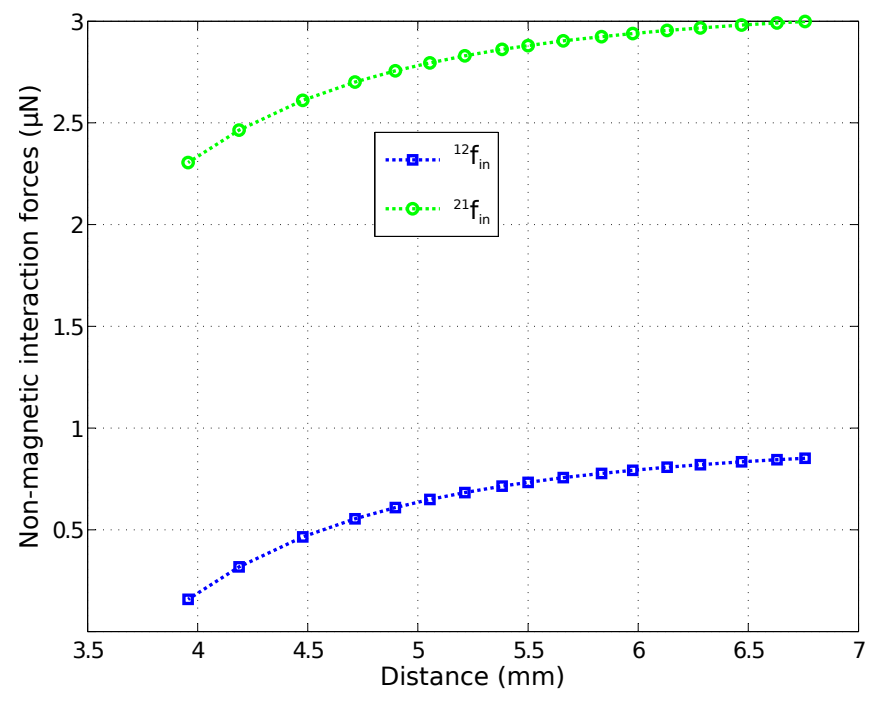

Figure 8. The experimental non magnetic interaction forces between boluses as function a separation distance.

\section{Discussions}

From the experimental results, it appears that the interaction force experienced on the bolus $B_{2}$ (the small one) due the presence of the bolus $B_{1}$ (the big one) is greater than the interaction force experienced by the bolus $B_{1}$ due to the presence of the bolus $B_{2}$. These difference are related to their shape and volume difference. Obviously, each magnetic bolus moves thanks to the magnetic interaction, but experience different drag forces $\mathbf{f}_{\mathrm{d} 1}$ and $\mathbf{f}_{\mathrm{d} 2}$ that are related to their shape. Secondly, the non-magnetic interaction forces are mainly related to the presence of hydrodynamic gradient pressure [26]. Actually, the motion of the magnetic bolus $B_{1}$ induce a high gradient pressure around the bolus $B_{2}$ which generates a noncontact forces. This gradient pressure is much higher around the bolus $B_{2}$, than in the vicinity of the bolus $B_{1}$.

\section{CONCLUSION}

This paper described the theoretical formulation of the magnetic interaction forces between two boluses. From experiments, we have estimated the magnetic and non magnetic interaction forces. This estimation show that the boluses experienced another interaction forces than magnetic interactions. The non magnetic forces is in micro newtons order and are not negligible. These forces are caused by the presence of a high gradient pressure around the bolus. When the boluses differ in the size, the forces experienced on the one bolus due to the presence of another bolus differ. In the future work, we develop a robust control law in order to control the motion of the boluses with the presence of the interaction forces.

\section{REFERENCES}

[1] T. Xu, J. Yu, X. Yan, H. Choi, and L. Zhang, "Magnetic actuation based motion control for microrobots: An overview," Micromachines, vol. 6, no. 9, pp. 1346-1364, 2015.

[2] L. Dong and B. J. Nelson, "Tutorial-robotics in the small part ii: nanorobotics," Robotics \& Automation Magazine, IEEE, vol. 14, no. 3, pp. 111-121, 2007.

[3] M. Sitti, H. Ceylan, W. Hu, J. Giltinan, M. Turan, S. Yim, and E. Diller, "Biomedical applications of untethered mobile milli/microrobots," Proceedings of the IEEE, vol. 103, no. 2, pp. 205-224, 2015.

[4] B. J. Nelson, I. K. Kaliakatsos, and J. J. Abbott, "Microrobots for minimally invasive medicine," Annual review of biomedical engineering, vol. 12, pp. 55-85, 2010.

[5] L. Zhang, T. Petit, K. E. Peyer, and B. J. Nelson, "Targeted cargo delivery using a rotating nickel nanowire," Nanomedicine: Nanotechnology, Biology and Medicine, vol. 8, no. 7, pp. 1074-1080, 2012.

[6] X. Yan, Q. Zhou, J. Yu, T. Xu, Y. Deng, T. Tang, Q. Feng, L. Bian, Y. Zhang, A. Ferreira et al., "Magnetite nanostructured porous hollow helical microswimmers for targeted delivery," Advanced Functional Materials, vol. 25, no. 33, pp. 5333-5342, 2015.

[7] S. Sanchez, A. A. Solovev, S. M. Harazim, and O. G. Schmidt, "Microbots swimming in the flowing streams of microfluidic channels," Journal of the American Chemical Society, vol. 133, no. 4, pp. 701-703, 2010.

[8] P. Pouponneau, J.-C. Leroux, G. Soulez, L. Gaboury, and S. Martel, "Coencapsulation of magnetic nanoparticles and doxorubicin into biodegradable microcarriers for deep tissue targeting by vascular mri navigation," Biomaterials, vol. 32, no. 13, pp. 3481-3486, 2011.

[9] P. Pouponneau, G. Bringout, and S. Martel, "Therapeutic magnetic microcarriers guided by magnetic resonance navigation for enhanced liver chemoembilization: A design review," Annals of biomedical engineering, vol. 42, no. 5, pp. 929-939, 2014.

[10] K. B. Yesin, K. Vollmers, and B. J. Nelson, "Modeling and control of untethered biomicrorobots in a fluidic environment using electromagnetic fields," The International Journal of Robotics Research, vol. 25, no. 5-6, pp. 527-536, 2006.

[11] L. Zhang, J. J. Abbott, L. Dong, B. E. Kratochvil, D. Bell, and B. J. Nelson, "Artificial bacterial flagella: Fabrication and magnetic control," Applied Physics Letters, vol. 94, no. 6, p. 064107, 2009.

[12] K. Belharet, D. Folio, and A. Ferreira, "Simulation and planning of a magnetically actuated microrobot navigating in the arteries," IEEE Trans. Biomed. Eng., vol. 60, no. 4, pp. 994-1001, 2013.

[13] A. Bigot, C. Tremblay, G. Soulez, and S. Martel, "Magnetic resonance navigation of a bead inside a three-bifurcation pmma phantom using an imaging gradient coil insert," Robotics, IEEE Transactions on, vol. 30, no. 3, pp. 719-727, 2014.

[14] S. Basak, D. Brogan, H. Dietrich, R. Ritter, R. G. Dacey, and P. Biswas, "Transport characteristics of nanoparticle-based ferrofluids in a gel model of the brain." International journal of nanomedicine, vol. 4, pp. 9-26, 2008.

[15] H. Nemala, A. Wadehra, A. Dixit, R. Regmi, P. Vaishnava, G. Lawes, and R. Naik, "Magnetic hyperthermia in ferrofluid-gel composites," in APS March Meeting Abstracts, vol. 1, 2012, p. 1194. 
[16] J.-B. Mathieu and S. Martel, "Magnetic microparticle steering within the constraints of an mri system: proof of concept of a novel targeting approach," Biomedical microdevices, vol. 9, no. 6, pp. 801-808, 2007.

[17] L. Mellal, K. Belharet, D. Folio, and A. Ferreira, "Optimal structure of particles-based superparamagnetic microrobots: application to MRI guided targeted drug therapy," J. of Nanoparticle Research, vol. 17, no. 2, pp. 1-18, 2015.

[18] A. Eqtami, O. Felfoul, and P. E. Dupont, "Mri-powered closed-loop control for multiple magnetic capsules," in Intelligent Robots and Systems (IROS 2014), 2014 IEEE/RSJ International Conference on. IEEE, 2014, pp. 3536-3542.

[19] E. Diller, S. Floyd, C. Pawashe, and M. Sitti, "Control of multiple heterogeneous magnetic microrobots in two dimensions on nonspecialized surfaces," Robotics, IEEE Transactions on, vol. 28, no. 1, pp. 172-182, 2012.

[20] L. D. Landau, J. Bell, M. Kearsley, L. Pitaevskii, E. Lifshitz, and J. Sykes, Electrodynamics of continuous media. elsevier, 1984, vol. 8.

[21] J. J. Abbott, O. Ergeneman, M. P. Kummer, A. M. Hirt, and B. J. Nelson, "Modeling magnetic torque and force for controlled manipulation of soft-magnetic bodies," Robotics, IEEE Transactions on, vol. 23, no. 6, pp. 1247-1252, 2007.

[22] R. A. Schill Jr, "General relation for the vector magnetic field of a circular current loop: a closer look," IEEE Transactions on Magnetics, vol. 39, no. 2, pp. 961-967, 2003.

[23] A. T. Becker, O. Felfoul, and P. E. Dupont, "Toward tissue penetration by mri-powered millirobots using a self-assembled gauss gun," in IEEE International Conference on Robotics and Automation (ICRA). IEEE, 2015, pp. 1184-1189.

[24] D. D. VILLANI, "An analytic solution for the force between two magnetic dipoles," Magnetic and electrical Separation, vol. 9, pp. 3952, 1998.

[25] K. Belharet, D. Folio, and A. Ferreira, "Control of a magnetic microrobot navigating in microfluidic arterial bifurcations through pulsatile and viscous flow," in IEEE/RSJ Int. Conf. on Intell. Robots and Syst. (IROS). IEEE, 2012, pp. 2559-2564.

[26] A. G. El-Gazzar, L. E. Al-Khouly, A. Klingner, S. Misra, and I. S. Khalil, "Non-contact manipulation of microbeads via pushing and pulling using magnetically controlled clusters of paramagnetic microparticles," in Intelligent Robots and Systems (IROS), 2015 IEEE/RSJ International Conference on. IEEE, 2015, pp. 778-783. 